\title{
Hematuria is a risk factor towards end-stage renal disease - A propensity score analysis
}

\author{
Tomoko Shima* \\ Department of Internal Medicine, Teikyo University School of Medicine, Tokyo, Japan
}

\begin{abstract}
Background: It remains unclear whether microscopic hematuria accelerates the progression of chronic kidney disease (CKD). Since the risk of microscopic hematuria was expected very weak, we employed a propensity score analysis.

Methods: A retrospective CKD cohort of 803 participants in a single institution was analyzed. The degree of microscopic hematuria was scaled as $0,0.5,1,2,3$ for negative,, $\pm 1+, 2+, 3+$ in the urinary qualitative analysis by dipstick, respectively. Time-averaged microscopic hematuria (TA-MH) was calculated by trapezoidal rule. The propensity score was estimated using 23 baseline covariates by multivariate binary logistic regression for the threshold of TA-MH at $\geq 0.5$ or $\geq 1.0$. Kaplan-Meier analysis after propensity score matching was also examined.

Results: The incidence rate of end-stage renal disease (ESRD) was 33.9 per 1,000 person-years over median follow-up of 4.3 years. A Cox regression analysis stratified by quintile on the propensity scores showed that TA-MH $\geq 0.5$ was a risk for ESRD (HR 1.72, 95\% CI 1.08-2.75, $p=0.023$ ) but not TA-MH $\geq 1.0$ (HR $1.34,95 \%$ CI $0.76-2.40, p=0.315)$. Kaplan-Meier analysis after propensity score matching reproduced the similar results $(\mathrm{TA}-\mathrm{MH} \geq 0.5, \mathrm{HR} 1.80,95 \% \mathrm{CI} 1.03-3.12, p=0.046$; TA-MH $\geq 1.0$, HR $1.73,95 \%$ CI $0.84-3.54, p=0.145)$.

Conclusions: Mild degree of microscopic hematuria is a risk factor for CKD progression to ESRD. The present result may help clinicians to recognize the CKD patients at higher risk due to the presence of microscopic hematuria.
\end{abstract}

\section{Introduction}

Early recognition and intervention against risk factors responsible for the progression of chronic kidney disease (CKD) are expected to improve renal outcomes of patients at risk [1]. Major risk factors of subsequent incidence of end-stage renal disease (ESRD) are hypertension, proteinuria and anemia in addition to preceding kidney dysfunction $[2,3]$. However, the second-line risk predictors remains to be clarified; candidates are hyperuricemia, hyperphosphatemia, metabolic acidosis, dyslipidemia, hypoalbuminemia, etc. Although hematuria has long been appreciated as a clinical presentation of the kidney diseases, it remains to be elucidated whether hematuria is a risk factor for progression of CKD. Recently, it has been suggested that young persons with persistent isolated microscopic hematuria may indeed have an increased risk for ESRD as compared with individuals without this condition [4]. On the other hand, there are several contradictory results, ruling out the impact of microscopic hematuria on the outcome of IgA nephropathy [5,6]. Therefore, microscopic hematuria has not reached consensus as a risk factor of ESRD [7].

The most probable reason for the conflicting results may lie on the small effect of microscopic hematuria compared with other major confounders such as proteinuria and hypertension. Thus, a propensity score analysis was employed, which is increasingly being used to estimate causal effects in the observational studies because one can replicate the prospective randomized controlled trial by minimizing baseline confounding [8]. Another important property long overlooked is the time-varying nature of microscopic hematuria in the clinical course of the CKD. In this study time-averaged microscopic hematuria (TA$\mathrm{MH}$ ) in the follow-up was calculated and used to investigate whether microscopic hematuria may be a risk factor of CKD progression.

\section{Patients and methods}

\section{Study protocol and ethical statement}

The retrospective CKD cohort already reported $(n=803)$ was used [9-12], and in the current study the propensity score analysis was used in such a way as used in the previously published articles $[9,12]$. Inclusion criteria consisted of CKD stage 3 to 4 , age 20 to 84 years and follow-up period $\geq 1$ year. On the other hand, patients with nephrotic syndrome, malignancy, obstructive nephropathy, acute kidney injury and gout were excluded. All the patients were followed up to 6 years until censoring or reaching the initiation of dialysis. The present study was approved by the institutional review board (IRB) in the Teikyo University Review Board \#14-115 and was executed in accordance with the principle of the Helsinki Declaration. Written informed consent was waived after approval of IRB and the patient records and information were anonymized and de-identified prior to analysis.

\section{Parameters analyzed}

The demographic characteristics included sex, age, body mass index (BMI), original kidney diseases (diabetic nephropathy, chronic

Correspondence to: Tomoko Shima, Department of Internal Medicine, Teikyo University School of Medicine, 2-11-1 Kaga, Itabashi-ku, Tokyo 173-8606, Japan, Tel: +81-3-3964-1211 (Ext. 33128); Fax: +81-3-3964-8942

Key words: microscopic hematuria, time-averaged microscopic hematuria, chronic kidney disease, end-stage renal disease, propensity score analysis

Received: January 09, 2017; Accepted: February 06, 2017; Published: February 09,2017 
glomerulonephritis, nephrosclerosis and other diseases) and systolic blood pressure (SBP). Diabetic nephropathy was defined as treated with antidiabetic agents or as having a long history of the disease. Chronic glomerulonephritis was defined as having biopsy-proven diagnosis or as absence of diabetes mellitus or hypertension. Nephrosclerosis was defined as treated with antihypertensive agents or as having a long history of hypertension. Other disease included polycystic kidney disease, tubulointerstitial nephritis and undetermined causes of CKD.

Blood parameters included hemoglobin $(\mathrm{Hb})$, white blood cell (WBC), platelet (Plt), albumin (Alb), uric acid (UA), sodium $(\mathrm{Na})$, potassium $(\mathrm{K})$, chloride $(\mathrm{Cl}), \mathrm{Na}-\mathrm{Cl}$ (as a surrogate of $\mathrm{HCO}_{3}$ ), albumin-corrected calcium ( $\mathrm{cCa}$ ), inorganic phosphorus $(\mathrm{P})$, lowdensity lipoprotein cholesterol (LDL-C) and C-reactive protein (CRP). Urine parameters included spot urine proteinuria (expressed as gram per gram creatinine excretion) and spot urine hematuria by dipstick (coded as four grades of 0 to 3 according to $0,1+, 2+$, and $3+$ and as 0.5 if \pm ). Due to the distribution, C-reactive protein and proteinuria were log-transformed for analyses.

Blood was tested using hematology autoanalyzer (Sysmex XE5000 , Kobe, Japan) and blood chemistry parameters were measured by routine measurements using autoanalyzer (LABOSPECT 008, Hitachi High-Technologies Corporation, Tokyo, Japan). Creatinine concentration in serum and urine was measured by an enzymatic method and urinary protein concentration measured by a pyrocatechol violet-metal complex assay method. Hematuria measured at every visit was recorded until censoring or reaching estimated GFR $5 \mathrm{~mL} / \mathrm{min} / 1.73$ $\mathrm{m}^{2}$ and calculated as TA-MH by trapezoidal rule $[9,12]$. Estimated GFR was evaluated using the Modification of Diet in Renal Disease (MDRD) study equation for Japanese population [13]. The grade of CKD was classified based on the Kidney Disease Outcomes Quality Initiative (K/ DOQI) practice guidelines [1].

Use of angiotensin converting enzyme inhibitor or angiotensin II receptor blocker (combined as RASi) and use of antiplatelet drugs were recorded as yes (coded as 1$)$ or no (coded as 0$)$. The baseline covariates used for the propensity score estimate modeling became 23 in total.

\section{Endpoints of renal outcomes}

A primary endpoint was defined as the incidence of ESRD (initiation of hemodialysis or peritoneal dialysis). Death was treated as censoring because the present study focused on the effect of hematuria on the subsequent ESRD rather than the risk of mortality [11]. Competing risk method was not employed due to a small portion of death censoring $(\mathrm{n}=10)$.

\section{A propensity score analysis}

The threshold of TA-MH in the follow-up was tested at 0.5 and 1.0. The probability to reach above or equal to the threshold was determined by a multivariate binary logistic regression using the aforementioned 23 baseline covariates. Since the distribution of propensity score of two groups differed widely, patients whose propensity scores not overlapped between two groups were trimmed, then the remaining subsample was re-stratified on the quintiles of the propensity scores $[14,15]$. A stratified Cox proportional hazards model was conducted in the substrata on the quintiles of the propensity scores [8]. Then, a pooled hazard ratio (HR) of the higher group of TA-MH was obtained as a crude HR.

Participants separated by the threshold of TA-MH (0.5 and 1.0) were matched using a greedy method with a 1:1 pair. The caliper size was first set at 0.25 times standard deviation of the propensity scores and gradually narrowed down until the balance of baseline 23 covariates were achieved between 2 groups [8]. The model of assignment was estimated by c-statistics and the balance between two groups was checked by paired comparison tests [16]. A time-to-event survival analysis was examined by a Kaplan-Meier analysis with stratified logrank test $[8,17]$.

\section{Statistical analyses}

Values for categorical variables are given as number (percentage) and values for continuous variables are given as mean \pm standard deviation or median [interquartile range] depending on the distribution. C-statistics for the accuracy of the propensity score models were obtained by the area under the receiver operating characteristic (AUROC) curve for the threshold [18]. For a Cox proportional hazards model, any covariate was tested for its proportional hazards assumption using both a time-dependent Cox regression and a Schoenfeld residual plot. Stratified log-rank test following Kaplan-Meier analysis after propensity score matching was performed by stratification on quintile of the matched subsample. Statistical analyses were performed using SPSS version 22 (IBM, Tokyo) and STATA version 14 (StataCorp LP, College Station, TX, USA). A $p$ value less than 0.05 was considered statistically significant.

\section{Results}

\section{Clinical characteristics of the cohort}

During the follow-up period of median 4.3 [interquartile range 2.65.7] years, 110 out of 803 patients progressed to ESRD. The incidence rate was 33.9 per 1,000 person-years. The patients $(n=236)$ having mild degree of hematuria ( $\mathrm{TA}-\mathrm{MH} \geq 0.5)$ in the follow-up showed the following baseline characteristics as shown Table 1. Estimated GFR was lower and chronic glomerulonephritis was more prevalent. Hemoglobin and serum albumin were lower and serum uric acid and serum phosphorus were higher. In addition, proteinuria and microscopic hematuria were greater in degree. The patients $(n=108)$ having greater degree of hematuria (TA-MH $\geq 1.0$ ) in the follow-up showed the following baseline characteristics as shown Table 2. Chronic glomerulonephritis was more prevalent but diabetic nephropathy less prevalent. Hemoglobin and serum albumin were lower while serum uric acid and serum phosphorus were higher. Proteinuria and microscopic hematuria were also greater.

Taken altogether, the patients presenting greater degree of microscopic hematuria, either 0.5 or 1.0 in the follow-up, were confounded with established risk factors such as anemia, hypoalbuminuria, hyperuricemia, hyperphosphatemia and proteinuria. It is no way questionable that hematuria did not show an independent risk factor by standard Cox regression analysis [9] (supplementary information).

\section{A propensity score analysis}

Stratified Cox proportional hazards model: A propensity score analysis could overcome the aforementioned problem. The threshold of TA-MH was examined at 0.5 and 1.0. A subsample after trimming nonoverlapped patients was re-stratified on the quintiles of the propensity scores and then subjected to survival analysis by a stratified Cox proportional hazards model. Proportional hazards assumption was not violated and multicollinearity was not seen. The result disclosed that the significantly high HR was found at 0.5 of the threshold of TA-MH (HR 1.72, 95\% CI 1.08-2.75, $p=0.023$ ). On the other hand, there was no 
statistical significance when $\mathrm{TA}-\mathrm{MH} \geq 1.0$ was tested.

Propensity score matching: Following propensity score matching, all the baseline covariates were well balanced by paired analysis when TA-MH 0.5 and 1.0 was used, respectively (Table 1 and 2). C-statistics estimated by the area under the ROC curve were all greater than 0.8 (Table 4), suggesting the high discrimination accuracy [16]. Then, two groups divided by the threshold of TA-MH were subjected to a KaplanMeier analysis (Table 4) that were plotted in Figure 1a and 1b. The patients showing TA-MH $\geq 0.5$ in the follow-up showed significantly higher HR for ESRD (HR 1.80, 95\%CI 1.03-3.12, $p=0.046$ ), whereas the threshold of TA-MH $\geq 1.0$ did not reach statistical significance (Table 4).

\section{Discussion}

In the present study we could show the significant impact of microscopic hematuria in the follow-up on the subsequent incidence of ESRD by applying the propensity score analysis. A pooled crude $\mathrm{HR}$ of TA-MH greater than or equal to 0.5 versus the lower group was significantly as high as 1.72 . To our astonishment, the target value of hematuria in the follow-up should be below \pm in dipstick test. The present study is the first report elucidating that even the mild hematuria in the follow-up underscores a risk factor of CKD progression, which was elucidated by applying the propensity score analysis.

Strength of the present study is two folds; calculation of TA-MH in the follow-up and the propensity score analysis. In most previous reports, hematuria was tested only once at baseline. Microscopic hematuria is likely to change with a clinical course of any types of $\mathrm{CKD}$, regarded as one of the time-varying parameters. Thus, TA-MH is better for assessing the impact of microscopic hematuria on CKD progression. In practice, arithmetically calculated time-averaged values have been utilized in time-to-event survival analyses [19-22]. Meanwhile, a propensity score analysis has come into rapid use in the clinical medicine because it can approximate randomized controlled trials using retrospective observational cohorts. The method also enables one to investigate the causal effect which cannot be otherwise executed due to serious issues such as ethical problems and inability of intervention. Propensity score analysis by use of time-averaged values of test parameters has a great advantage to freely scrutinize the threshold of target values as demonstrated here and previously [9].

Although microscopic hematuria has been considered as a diagnostic marker of any kidney disease including glomerular diseases, its impact on CKD progression has not been investigated in many large

Table 1. Balance of baseline covariates before and after propensity score matching for TA-MH 0.5 .

\begin{tabular}{|c|c|c|c|c|c|c|}
\hline \multirow[t]{2}{*}{ Characteristics } & \multicolumn{4}{|c|}{ Before matching $(n=803)$} & \multicolumn{2}{|c|}{ After matching $(n=270)$} \\
\hline & $\begin{array}{c}\text { TA-MH }<0.5 \\
n=567\end{array}$ & $\begin{array}{c}\text { TA-MH } \geq 0.5 \\
n=236\end{array}$ & $p$ value ${ }^{*}$ & $\begin{array}{c}\text { TA-MH }<0.5 \\
n=135\end{array}$ & $\begin{array}{c}\text { TA-UA } \geq 0.5 \\
\quad n=135\end{array}$ & $p$ value $^{\dagger}$ \\
\hline TA-MH & $0.12 \pm 0.14$ & $1.09 \pm 0.58$ & $<0.001$ & $0.18 \pm 0.14$ & $0.89 \pm 0.42$ & $<0.001$ \\
\hline Age (y) & $62.6 \pm 12.5$ & $61.1 \pm 14.3$ & 0.139 & $63.7 \pm 12.1$ & $62.3 \pm 14.0$ & 0.353 \\
\hline eGFR $\left(\mathrm{mL} / \mathrm{min} / 1.73 \mathrm{~m}^{2}\right)$ & $41.8 \pm 12.9$ & $39.5 \pm 13.8$ & 0.024 & $41.3 \pm 12.9$ & $40.5 \pm 14.5$ & 0.620 \\
\hline Male (\%) & $360(63.5)$ & $141(59.7)$ & 0.338 & $69(51.1)$ & $80(53.7)$ & 0.145 \\
\hline DMN (\%) & $132(23.3)$ & $54(22.9)$ & 0.927 & $37(27.4)$ & $28(20.7)$ & 0.225 \\
\hline CGN (\%) & $112(19.8)$ & $64(27.1)$ & 0.025 & $33(24.4)$ & $33(24.4)$ & 1.000 \\
\hline $\operatorname{BMI}\left(\mathrm{kg} / \mathrm{m}^{2}\right)$ & $24.4 \pm 4.3$ & $24.2 \pm 4.5$ & 0.454 & $24.5 \pm 4.4$ & $24.5 \pm 4.8$ & 0.944 \\
\hline SBP (mmHg) & $137.7 \pm 20.6$ & $136.4 \pm 21.8$ & 0.426 & $137.3 \pm 19.5$ & $135.7 \pm 21.4$ & 0.509 \\
\hline \multicolumn{7}{|l|}{ Blood } \\
\hline $\mathrm{Hb}(\mathrm{g} / \mathrm{dL})$ & $13.0 \pm 1.9$ & $12.6 \pm 2.0$ & 0.015 & $12.7 \pm 1.8$ & $12.6 \pm 2.0$ & 0.786 \\
\hline $\mathrm{WBC}\left(\times 10^{2} / \mu \mathrm{L}\right)$ & $64.7 \pm 21.3$ & $67.6 \pm 21.7$ & 0.078 & $65.7 \pm 23.4$ & $66.2 \pm 21.3$ & 0.879 \\
\hline Plt $\left(\times 10^{4} / \mu \mathrm{L}\right)$ & $21.9 \pm 6.4$ & $22.2 \pm 7.7$ & 0.515 & $21.6 \pm 6.2$ & $21.8 \pm 6.6$ & 0.792 \\
\hline $\mathrm{Alb}(\mathrm{g} / \mathrm{dL})$ & $4.0 \pm 0.5$ & $3.8 \pm 0.5$ & $<0.001$ & $3.8 \pm 0.5$ & $3.9 \pm 0.5$ & 0.176 \\
\hline $\mathrm{UA}(\mathrm{mg} / \mathrm{dL})$ & $6.4 \pm 1.4$ & $6.7 \pm 1.4$ & 0.020 & $6.5 \pm 1.5$ & $6.6 \pm 1.5$ & 0.602 \\
\hline $\mathrm{Na}(\mathrm{mEq} / \mathrm{L})$ & $140.8 \pm 2.7$ & $140.7 \pm 2.6$ & 0.682 & $140.7 \pm 2.4$ & $140.7 \pm 2.8$ & 0.818 \\
\hline $\mathrm{K}(\mathrm{mEq} / \mathrm{L})$ & $4.5 \pm 0.5$ & $4.5 \pm 0.6$ & 0.759 & $4.5 \pm 0.5$ & $4.5 \pm 0.6$ & 0.875 \\
\hline $\mathrm{Na}-\mathrm{Cl}(\mathrm{mEq} / \mathrm{L})$ & $35.5 \pm 2.4$ & $35.1 \pm 2.7$ & 0.053 & $35.2 \pm 2.1$ & $35.2 \pm 2.7$ & 0.961 \\
\hline $\mathrm{cCa}(\mathrm{mg} / \mathrm{dL})$ & $8.8 \pm 0.5$ & $8.9 \pm 0.5$ & 0.009 & $9.0 \pm 0.5$ & $8.9 \pm 0.5$ & 0.375 \\
\hline$P(\mathrm{mg} / \mathrm{dL})$ & $3.3 \pm 0.5$ & $3.5 \pm 0.6$ & $<0.001$ & $3.4 \pm 0.5$ & $3.5 \pm 0.6$ & 0.455 \\
\hline CRP (mg/dL) & $0.08[0.04-0.18]$ & $0.10[0.05-0.24]$ & 0.045 & $0.08[0.05-0.17]$ & $0.10[0.05-0.24]$ & 0.581 \\
\hline LDL-C (mg/dL) & $112.2 \pm 31.0$ & $107.6 \pm 29.0$ & 0.050 & $107.8 \pm 30.7$ & $106.8 \pm 29.4$ & 0.798 \\
\hline \multicolumn{7}{|l|}{ Spot urine } \\
\hline TPU/CrU (g/g Cr) & $0.36[0.16-0.99]$ & $0.73[0.25-1.75]$ & $<0.001$ & $0.49[0.22-1.55]$ & $0.48[0.19-1.36]$ & 0.496 \\
\hline UB_score & $0.22 \pm 0.47$ & $1.27 \pm 1.00$ & $<0.001$ & $0.63 \pm 0.76$ & $0.67 \pm 0.69$ & 0.123 \\
\hline \multicolumn{7}{|l|}{ Drug use } \\
\hline RASi, Yes (\%) & $319(56.3)$ & $118(50.0)$ & 0.120 & $80(59.3)$ & $73(54.1)$ & 0.406 \\
\hline Antiplatelet drugs, Yes (\%) & $60(10.6)$ & $25(10.6)$ & 1.000 & $11(8.1)$ & $16(11.9)$ & 0.297 \\
\hline
\end{tabular}

Note: Values for categorical variables are given as number (percentage); values for continuous variables are given as mean \pm standard deviation or median [interquartile range]. For statistical analyses, CRP and TPU/CrU were log-transformed. Conversion factors for units: creatinine in $\mathrm{mg} / \mathrm{dL}$ to $\mu \mathrm{mol} / \mathrm{L}, \mathrm{x} 88.4$; uric acid in $\mathrm{mg} / \mathrm{dL}$ to $\mu \mathrm{mol} / \mathrm{L}$, $\mathrm{x} 59.48$.

*Unpaired $t$ test or chi square test

†Paired $t$ test or McNemar test

Abbreviations: TA-MH, time-averaged microscopic hematuria; eGFR, estimated glomerular filtration rate; DMN, diabetic nephropathy; CGN, chronic glomerulonephritis; BMI, Body Mass Index; SBP, systolic blood pressure; Hb, hemoglobin; WBC, white blood cells; Plt, Platelet; Alb, albumin; UA, uric acid; Na, sodium; K, potassium; Cl, chloride; cCa, albumin-corrected calcium; P, phosphorus; LDL-C, low-density lipoprotein cholesterol; TPU/CrU, urine total protein divided by urine creatinine; UB_score, urine blood score; RASi, RAS inhibitor. 
Table 2. Balance of baseline covariates before and after propensity score matching by TA-MH 1.0.

\begin{tabular}{|c|c|c|c|c|c|c|}
\hline \multirow[t]{2}{*}{ Characteristics } & \multicolumn{3}{|c|}{ Before matching $(n=803)$} & \multicolumn{3}{|c|}{ After matching $(\mathrm{n}=\mathbf{1 5 0})$} \\
\hline & $\begin{array}{c}\text { TA-MH }<1 \\
\text { n }=695\end{array}$ & $\begin{array}{c}\text { TA-MH } \geq 1 \\
\mathrm{n}=108\end{array}$ & $p$ value $^{*}$ & $\begin{array}{c}\text { TA-MH }<1 \\
n=75\end{array}$ & $\begin{array}{c}\text { TA-UA } \geq 1 \\
n=75\end{array}$ & $p$ value $^{\dagger}$ \\
\hline TA-MH & $0.22 \pm 0.26$ & $1.58 \pm 0.52$ & $<0.001$ & $0.43 \pm 0.31$ & $1.45 \pm 0.43$ & $<0.001$ \\
\hline Age (y) & $62.4 \pm 12.7$ & $60.4 \pm 15.2$ & 0.126 & $62.0 \pm 14.1$ & $61.2 \pm 15.3$ & 0.740 \\
\hline eGFR $\left(\mathrm{mL} / \mathrm{min} / 1.73 \mathrm{~m}^{2}\right)$ & $41.1 \pm 13.1$ & $41.3 \pm 13.3$ & 0.889 & $38.9 \pm 13.0$ & $39.3 \pm 13.9$ & 0.862 \\
\hline Male (\%) & $442(63.6)$ & $59(54.6)$ & 0.087 & $38(50.7)$ & $41(54.7)$ & 0.749 \\
\hline DMN (\%) & $171(24.6)$ & $15(13.9)$ & 0.014 & $16(21.3)$ & $14(18.7)$ & 0.845 \\
\hline CGN (\%) & $139(20.0)$ & $37(34.3)$ & 0.002 & $18(24.0)$ & $22(29.3)$ & 0.580 \\
\hline $\operatorname{BMI}\left(\mathrm{kg} / \mathrm{m}^{2}\right)$ & $24.5 \pm 4.3$ & $23.5 \pm 4.4$ & 0.035 & $24.3 \pm 4.4$ & $24.2 \pm 4.4$ & 0.852 \\
\hline SBP $(\mathrm{mmHg})$ & $137.8 \pm 20.9$ & $134.0 \pm 20.7$ & 0.075 & $137.4 \pm 20.0$ & $136.6 \pm 19.4$ & 0.815 \\
\hline \multicolumn{7}{|l|}{ Blood Parameters } \\
\hline $\mathrm{Hb}(\mathrm{g} / \mathrm{dL})$ & $12.9 \pm 1.9$ & $12.3 \pm 2.0$ & 0.002 & $12.4 \pm 2.1$ & $12.2 \pm 2.1$ & 0.688 \\
\hline $\mathrm{WBC}\left(\times 10^{2} / \mu \mathrm{L}\right)$ & $65.3 \pm 21.1$ & $66.9 \pm 23.3$ & 0.473 & $65.7 \pm 22.0$ & $66.3 \pm 21.8$ & 0.881 \\
\hline Plt $\left(\times 10^{4} / \mu \mathrm{L}\right)$ & $22.0 \pm 6.6$ & $22.1 \pm 8.1$ & 0.861 & $22.0 \pm 8.0$ & $22.6 \pm 8.6$ & 0.652 \\
\hline $\mathrm{Alb}(\mathrm{g} / \mathrm{dL})$ & $4.0 \pm 0.5$ & $3.8 \pm 0.5$ & $<0.001$ & $3.8 \pm 0.4$ & $3.8 \pm 0.5$ & 0.769 \\
\hline $\mathrm{UA}(\mathrm{mg} / \mathrm{dL})$ & $6.4 \pm 1.4$ & $6.8 \pm 1.5$ & 0.016 & $6.6 \pm 1.4$ & $6.8 \pm 1.6$ & 0.492 \\
\hline $\mathrm{Na}(\mathrm{mEq} / \mathrm{L})$ & $140.7 \pm 2.7$ & $140.9 \pm 2.3$ & 0.586 & $140.6 \pm 2.9$ & $140.8 \pm 2.4$ & 0.644 \\
\hline $\mathrm{K}(\mathrm{mEq} / \mathrm{L})$ & $4.5 \pm 0.5$ & $4.5 \pm 0.6$ & 0.727 & $4.6 \pm 0.6$ & $4.5 \pm 0.5$ & 0.588 \\
\hline $\mathrm{Na}-\mathrm{Cl}(\mathrm{mEq} / \mathrm{L})$ & $35.4 \pm 2.4$ & $35.0 \pm 2.9$ & 0.113 & $35.0 \pm 2.4$ & $34.8 \pm 3.1$ & 0.672 \\
\hline $\mathrm{cCa}(\mathrm{mg} / \mathrm{dL})$ & $8.8 \pm 0.5$ & $8.9 \pm 0.4$ & 0.250 & $8.9 \pm 0.5$ & $8.9 \pm 0.4$ & 0.752 \\
\hline $\mathrm{P}(\mathrm{mg} / \mathrm{dL})$ & $3.4 \pm 0.5$ & $3.5 \pm 0.6$ & 0.038 & $3.4 \pm 0.4$ & $3.5 \pm 0.6$ & 0.512 \\
\hline $\mathrm{CRP}(\mathrm{mg} / \mathrm{dL})$ & $0.08[0.05-0.20]$ & $0.10[0.05-0.22]$ & 0.497 & $0.07[0.05-0.17]$ & $0.10[0.04-0.23]$ & 0.450 \\
\hline LDL-C (mg/dL) & $111.5 \pm 31.1$ & $106.6 \pm 26.2$ & 0.116 & $109.4 \pm 31.2$ & $109.4 \pm 26.0$ & 0.998 \\
\hline \multicolumn{7}{|l|}{ Spot urine } \\
\hline TPU/CrU (g/g Cr) & $0.40[0.17-1.09]$ & $0.73[0.27-1.64]$ & $<0.001$ & $0.78[0.22-1.64]$ & $0.66[0.25-1.48]$ & 0.899 \\
\hline UB_score & $0.34 \pm 0.61$ & $1.74 \pm 0.97$ & $<0.001$ & $1.39 \pm 1.06$ & $1.41 \pm 0.94$ & 0.886 \\
\hline \multicolumn{7}{|l|}{ Drug use } \\
\hline RASi, Yes (\%) & $387(55.7)$ & $50(46.3)$ & 0.077 & $38(50.7)$ & $35(46.7)$ & 0.749 \\
\hline Antiplatelet drugs, Yes (\%) & $74(10.6)$ & $11(10.2)$ & 1.000 & $7(9.3)$ & $7(9.3)$ & 1.000 \\
\hline
\end{tabular}

Note: Values for categorical variables are given as number (percentage); values for continuous variables are given as mean \pm standard deviation or median [interquartile range]. For statistical analyses, CRP and TPU/CrU were log-transformed. Conversion factors for units: creatinine in $\mathrm{mg} / \mathrm{dL}$ to $\mu \mathrm{mol} / \mathrm{L}, \mathrm{x} 88.4$; uric acid in $\mathrm{mg} / \mathrm{dL}$ to $\mu \mathrm{mol} / \mathrm{L}$, $\mathrm{x} 59.48$.

*Unpaired $t$ test or chi square test

†Paired $t$ test or McNemar test

Abbreviations: TA-MH, time-averaged microscopic hematuria; eGFR, estimated glomerular filtration rate; DMN, diabetic nephropathy; CGN, chronic glomerulonephritis; BMI, Body Mass Index; SBP, systolic blood pressure; Hb, hemoglobin; WBC, white blood cells; Plt, Platelet; Alb, albumin; UA, uric acid; Na, sodium; K, potassium; Cl, chloride; cCa, albumin-corrected calcium; P, phosphorus; LDL-C, low-density lipoprotein cholesterol; TPU/CrU, urine total protein divided by urine creatinine; UB_score, urine blood score; RASi, RAS inhibitor.

Table 3. Stratified Cox regression for ESRD by thresholds of TA-MH.

\begin{tabular}{|c|c|c|c|}
\hline Threshold of TA-MH & $\begin{array}{c}\text { Patients after trimming } \\
\text { (Total/Incident) }\end{array}$ & HR & 95\% CI \\
\hline$\geq 0.5$ & $765 / 108$ & 1.72 & $1.08-2.75$ \\
\hline$\geq 1.0$ & $717 / 96$ & 1.34 & 0.023 \\
\hline
\end{tabular}

Abbreviations: ESRD, end-stage renal disease; TA-MH, time-averaged microscopic hematuria; HR, hazard ration; CI, confidence interval

Table 4. Kaplan-Meier analysis after propensity score matching.

\begin{tabular}{|l|l|c|c|c|c|c|c|}
\hline \multirow{2}{*}{$\begin{array}{l}\text { Threshold of TA- C-statistics } \\
\text { MH }\end{array}$} & \multicolumn{2}{|c|}{ Before matching } & \multicolumn{2}{|c|}{ After matching } \\
\cline { 2 - 7 } & & $\begin{array}{c}\text { Low group } \\
\text { (Total/Incident) }\end{array}$ & $\begin{array}{c}\text { High group } \\
\text { (Total/Incident) }\end{array}$ & $\begin{array}{c}\text { Low group } \\
\text { (Total/Incident) }\end{array}$ & $\begin{array}{c}\text { High group } \\
\text { (Total/Incident) }\end{array}$ & $\begin{array}{c}\text { HR } \\
\text { (stratified log-rank) }\end{array}$ & \begin{tabular}{c} 
95\% CI \\
\hline 0.5
\end{tabular} \\
\hline 1 & 0.86 & $567 / 55$ & $236 / 55$ & $135 / 21$ & 1.80 & $1.03-3.12$ & 0.046 \\
\hline
\end{tabular}

Abbreviations: TA-MH, time-averaged microscopic hematuria; HR, hazard ratio; CI, confidence interval

epidemiological studies $[23,24]$. This fact may be in part due to the difficulty in assessing microscopic hematuria quantitatively $[25,26]$. Recently, Vivante et al. reported that persistent asymptomatic isolated microscopic hematuria was significantly associated with increased risk of ESRD over 22 years of follow-up in 1.2 million persons aged 16 through 25 years [4]. This study suggests that hematuria in the absence of a specific diagnosis can be a risk factor of ESRD and the classical term "benign hematuria" or "benign familial hematuria" may be a misnomer, especially in most cases where neither a histopathologic nor a genetic diagnosis is made [4]. Goto et al. also showed that mild hematuria was associated with increased risk of ESRD over 10 years of follow-up in $790 \mathrm{IgA}$ nephropathy patients. Most recently, Lin et al. showed that microscopic hematuria was associated with increased risk of ESRD in 1,799 patients with stage 3 to 5 nondiabetic CKD. Whereas, there are several reports denying microscopic hematuria as a risk factor of CKD progression in patients with IgA nephropathy $[5,6]$ 


\section{a.}

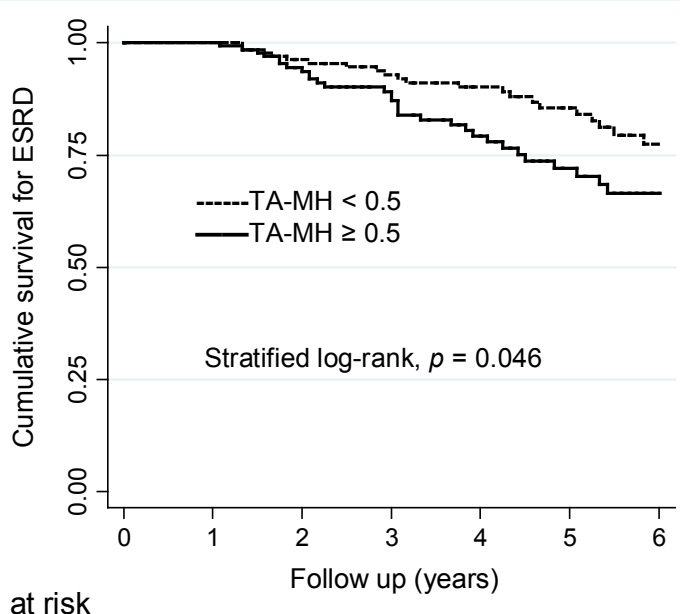

Patients at risk

Low TA-MH group 135

High TA-MH group 135

\section{2}

87

11

b.

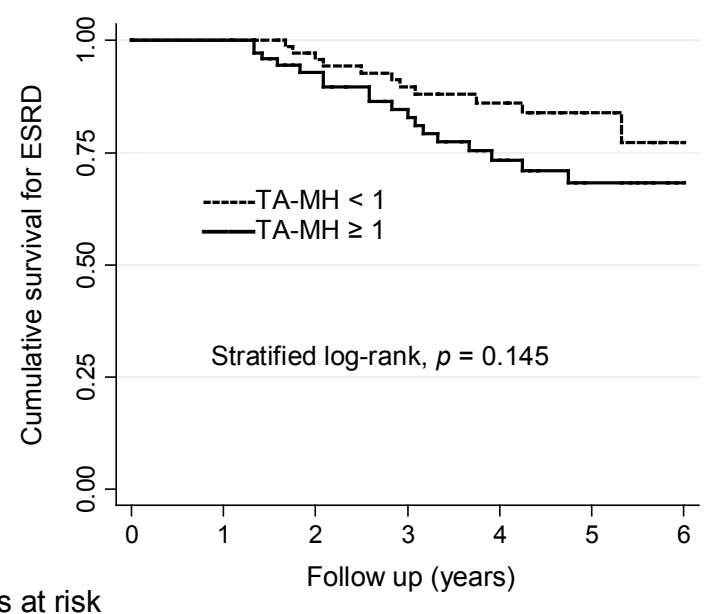

\section{Patients at risk}

Low TA-MH group 75

High TA-MH group 75

66

59

43

35

7

Figure 1. Kaplan-Meier plots after the propensity score matching. a)Time-averaged microscopic hematuria (TA-MH) $\geq 0.5$ and $<0.5, b$ ) TA-MH $\geq 1.0$ and $<1.0$. b) The patien numbers at risk are shown below and $\mathrm{p}$ values are estimated by stratified log-rank test.

and ANCA vasculitis [27]. Therefore, it remains to be inconclusive whether microscopic hematuria is a risk factor of CKD progression. In the present study, mild hematuria was significantly associated with increased risk of ESRD after employing propensity score analysis. This fact suggested that mild hematuria is a good prognostic marker in CKD patients. However, the lack of dose response effect of microscopic hematuria has to be emphasized. One possible reason may lie on the decrease in the patient numbers both in stratified Cox regression analysis and Kaplan-Meier analysis. An alternative mechanism is that the effect of microscopic hematuria on CKD progression is saturated until when it represents $1+$ or greater in dipstick test. This interesting and important question warrants further investigation.
Hematuria could induce renal tubular epithelial cell damage by the cytotoxic, oxidant and inflammatory effects of hemoglobin-related molecules such as heme and iron [28]. Erythrocyte passage across the glomerular filtration barrier induces mechanical stress to erythrocyte, leading to leakage of hemoglobin from erythrocyte. Tubular epithelial cells can uptake hemoglobin in the urinary space via magalin-cubulin receptor system. Hemoglobin is then transformed into heme and globin [29]. Free heme is extremely toxic, promoting lipid oxidation, inducing apoptosis and releasing inflammatory chemokines [30]. This heme-induced oxidation and inflammation in tubular cells could be related to CKD progression in the patients presenting microscopic hematuria [28]. Future studies are needed to elucidate the mechanism how hematuria exacerbates kidney damage in CKD milieu. In any event the present finding may open up a new horizon for the impact of hematuria on the progression of CKD.

Some limitations of the present study should be mentioned. The first limitation is that we assessed microscopic hematuria only by dipstick test but not by urinary sediment. So there is a possibility that false positive seen at myoglobinuria and false negative seen at use of ascorbic acid may exist. Next, in female patients there is the possibility that menstrual blood may come to be mixed in urine. Not with standing, the mean age of the cohort greater than 60 may negate this possibility. Thirdly, there is the potential presence of unmeasured confounders which cannot be avoided in any observational study. Lastly, there is a possibility of misspecification of the propensity score model which cannot be asserted by any means, either. Despite these limitations, a propensity score analysis clearly captures its unbounded potential to examine many test conditions such as risk factors and target thresholds. Particularly intervention is not easy to perform such as microscopic hematuria.

\section{Conclusion}

The propensity score analysis unveiled that mild hematuria in the follow-up can predict the risk of ESRD. Target range of hematuria in the follow-up is less than \pm in dipstick test to inhibit the progression of CKD to ESRD. The results may facilitate vigorous surveillance of microscopic hematuria which has been prone to be ignored in CKD practice. The mechanism whereby mild hematuria renders kidney injury in the setting of CKD should be elucidated in animal and human studies.

\section{Acknowledgments}

I cordially thank Professor Shunya Uchida, Division of Nephrology, Department of Internal Medicine, Teikyo University School of Medicine, for his continued support, encouragement, discussion and critical reading of the manuscript. I also thank Dr. Takanori Kumagai for help writing the draft and Dr. Wen Xiu Chang, Tianjin First Central Hospital, Tianjin, China, for superb help in statistical analysis, and all of the doctors at the Division of Nephrology, Department of Internal Medicine, Teikyo University School of Medicine, Tokyo, Japan, for their kind cooperation.

\section{Support}

This study was supported in part by a Grant-in-Aid for Progressive Renal Diseases Research, Research on Rare and Intractable Disease, from the Ministry of Health, Labour and Welfare of Japan (to SU). The funders had no role in study design, data collection and analysis, 
decision to publish, or preparation of the manuscript.

\section{Competing interests}

The author has declared that no competing interests exist.

\section{Funding}

The author received no specific funding for this work.

\section{References}

1. Inker LA, Astor BC, Fox CH, Isakova T, Lash JP, et al. (2014) KDOQI US commentary on the 2012 KDIGO clinical practice guideline for the evaluation and management of CKD. Am J Kidney Dis 63: 713-735. [Crossref]

2. Johnson ES, Smith DH, Thorp ML, Yang X, Juhaeri J (2011) Predicting the risk of endstage renal disease in the population-based setting: a retrospective case-control study. BMC Nephrol 12: 17. [Crossref]

3. Yang W, Xie D, Anderson AH, Joffe MM, Greene T, et al. (2014) Association of kidney disease outcomes with risk factors for CKD: findings from the Chronic Renal Insufficiency Cohort (CRIC) study. Am J Kidney Dis 63: 236-243. [Crossref]

4. Vivante A, Afek A, Frenkel-Nir Y, Tzur D, Farfel A, et al. (2011) Persistent asymptomatic isolated microscopic hematuria in Israeli adolescents and young adults and risk for end-stage renal disease. JAMA 306: 729-736.

5. Gutiérrez E, Zamora I, Ballarín JA, Arce Y, Jiménez S, et al. (2012) Long-term outcomes of IgA nephropathy presenting with minimal or no proteinuria. $J$ Am Soc Nephrol 23: 1753-1760. [Crossref]

6. Tanaka K, Moriyama T, Iwasaki C, Takei T, Nitta K (2015) Effect of hematuria on the outcome of IgA nephropathy with mild proteinuria. Clin Exp Nephrol 19: 815-821. [Crossref]

7. Moreno JA, Yuste C, Gutierrez E, Sevillano AM, Rubio-Navarro A, et al. (2016) Haematuria as a risk factor for chronic kidney disease progression in glomerular diseases: A review. Pediatr Nephrol 31: 523-533. [Crossref]

8. Austin PC (2014) The use of propensity score methods with survival or time-toevent outcomes: reporting measures of effect similar to those used in randomized experiments. Stat Med 33: 1242-1258. [Crossref]

9. Uchida S, Chang WX, Ota T, Tamura Y, Shiraishi T, et al. (2015) Targeting uric acid and the inhibition of progression to end-stage renal disease--a propensity score analysis. PLoS One 10: e0145506. [Crossref]

10. Chang WX, Arai S, Tamura Y, Kumagai T, et al. (2016) Time-dependent risk factors associated with the decline of estimated GFR in CKD patients. Clin Exp Nephrol 20 : 58-70. [Crossref]

11. Chang WX, Asakawa S, Toyoki D, Nemoto Y, Morimoto C, et al. (2015) Predictors and the subsequent risk of end-stage renal disease - usefulness of $30 \%$ decline in estimated GFR over 2 years. PLoS One 10: e0132927. [Crossref]

12. Chang WX, Xu N, Kumagai T, Shiraishi T, et al. (2016) The impact of normal range of serum phosphorus on the incidence of end-stage renal disease by a propensity score analysis. PLoS One 11: e0154469. [Crossref]

13. Matsuo S, Imai E, Horio M, Yasuda Y, Tomita K, et al. (2009) Revised equations for estimated GFR from serum creatinine in Japan. Am J Kidney Dis 53: 982-992. [Crossref]
14. Petersen ML, Porter KE, Gruber S, Wang Y, van der Laan MJ (2012) Diagnosing and responding to violations in the positivity assumption. Stat Methods Med Res 21:31-54. [Crossref]

15. Vansteelandt S, Daniel RM (2014) On regression adjustment for the propensity score. Stat Med 33: 4053-4072. [Crossref]

16. Uno H, Cai T, Pencina MJ, D'Agostino RB, Wei LJ (2011) On the C-statistics for evaluating overall adequacy of risk prediction procedures with censored survival data Stat Med 30: 1105-1117. [Crossref]

17. Austin PC (2008) A critical appraisal of propensity-score matching in the medical literature between 1996 and 2003. Stat Med 27: 2037-2049. [Crossref]

18. Normand ST, Landrum MB, Guadagnoli E, Ayanian JZ, Ryan TJ, et al. (2001) Validating recommendations for coronary angiography following acute myocardial infarction in the elderly: a matched analysis using propensity scores. J Clin Epidemiol 54: 387-398.

19. Reich HN, Troyanov S, Scholey JW, Cattran DC; Toronto Glomerulonephritis Registry (2007) Remission of proteinuria improves prognosis in IgA nephropathy. $J$ Am Soc Nephrol 18: 3177-3183. [Crossref]

20. Seiler S, Lucisano G, Ege P, Fell LH, Rogacev KS, et al. (2013) Single FGF-23 measurement and time-averaged plasma phosphate levels in hemodialysis patients Clin J Am Soc Nephrol 8: 1764-1772. [Crossref]

21. Bouma-de Krijger A, Bots ML, Vervloet MG, Blankestijn PJ, Ter Wee PW, et al (2014) Time-averaged level of fibroblast growth factor-23 and clinical events in chronic kidney disease. Nephrol Dial Transplant 29: 88-97. [Crossref]

22. Ni Z, Yuan Y, Wang Q, Cao L, Che X, et al. (2014) Time-averaged albumin predicts the long-term prognosis of IgA nephropathy patients who achieved remission. J Transl Med 12: 194. [Crossref]

23. Coresh J, Turin TC, Matsushita K, Sang Y, Ballew SH, et al. (2014) Decline in estimated glomerular filtration rate and subsequent risk of end-stage renal disease and mortality. JAMA 311: 2518-2531. [Crossref]

24. Khan UA, Garg AX, Parikh CR, Coca SG (2013) Prevention of chronic kidney disease and subsequent effect on mortality: a systematic review and meta-analysis. PLoS One 8: e71784. [Crossref]

25. Fogazzi GB, Verdesca S, Garigali G (2008) Urinalysis: core curriculum 2008. Am J Kidney Dis 51: 1052-1067. [Crossref]

26. Yuste C, Rubio-Navarro A, Barraca D, Aragoncillo I, Vega A, et al. (2015) Haematuria increases progression of advanced proteinuric kidney disease. PLoS One 10: 0128575. [Crossref]

27. Chen TK, Murakami C, Manno RL, Geetha D. (2014) Hematuria duration does not predict kidney function at 1 year in ANCA-associated glomerulonephritis. Semin Arthritis Rheum 44: 198-201.

28. Tracz MJ, Alam J, Nath KA (2007) Physiology and pathophysiology of heme: implications for kidney disease. $J$ Am Soc Nephrol 18: 414-420. [Crossref]

29. Moreno JA, Martín-Cleary C, Gutiérrez E, Rubio-Navarro A, Ortiz A, et al. (2012) Haematuria: the forgotten CKD factor? Nephrol Dial Transplant 27: 28-34. [Crossref]

30. Nath KA, Croatt AJ, Haggard JJ, Grande JP (2000) Renal response to repetitive exposure to heme proteins: chronic injury induced by an acute insult. Kidney Int 57 : 2423-2433. [Crossref]

Copyright: (C2017 Shima T. This is an open-access article distributed under the terms of the Creative Commons Attribution License, which permits unrestricted use, distribution, and reproduction in any medium, provided the original author and source are credited. 